Revista Brasileira de Agricultura Irrigada v.9, nº.3, p. 158 - 170, 2015

ISSN 1982-7679 (On-line)

Fortaleza, CE, INOVAGRI - http://www.inovagri.org.br

DOI: $10.7127 /$ rbai.v9n300301

Protocolo 301.15 - 10/03/2015 Aprovado em 08/05/2015

\title{
AVALIAÇÃO DO RISCO DE CONTAMINAÇÃO HÍDRICA POR AGROTÓXICOS NO PERÍMETRO IRRIGADO BETUME NO BAIXO RIO SÃO FRANCISCO
}

\author{
F. B. Britto ${ }^{1}$, T. M. M. da Silva ${ }^{2}$, A. N. do Vasco $^{3}$, A. O. Aguiar Netto ${ }^{4}$, C. M. de Carvalho 5
}

\section{RESUMO}

A crescente atividade agrícola no perímetro irrigado Betume tem causado preocupações quanto à contaminação por agrotóxico em áreas irrigadas. O problema se torna mais importante quando a contaminação pode afetar a água para o uso humano, como ocorre com a água da bacia hidrográfica do Rio Betume que é um afluente do Rio São Francisco, e abastece diversas regiões do estado de Sergipe. O objetivo do estudo foi avaliar o risco de contaminação de águas superficiais e subterrâneas por agrotóxico e monitorar a qualidade da água em áreas de rizicultura na bacia hidrográfica do rio Betume. O monitoramento foi realizado bimensalmente de março de 2013 a dezembro de 2014 e a análise de risco de contaminação foi realizada mediante critérios da Environmental Protection Agency (EPA), Índice de GUS e método de GOSS. Os resultados evidenciaram a presença dos seguintes princípios ativos: clorpirifós, tebuconazol e tetraconazol, em que a resolução CONAMA $n^{\circ}$ 357/05 não contemplam os limites de tolerância de agrotóxico na água para a proteção do meio ambiente referentes a estes produtos. Quando comparados com os padrões da Comunidade Europeia, as concentrações de tebuconazole e clorpirifós ficaram acima do limite de tolerância. A presença de agrotóxico na região evidencia os riscos de contaminação da população, sendo assim, as medidas de prevenção na manipulação e aplicação do agrotóxico devem ser tomadas para garantir a qualidade da água.

Palavras-chave: monitoramento ambiental, contaminação de águas superficiais e subterrâneas e rizicultura.

\section{CONTAMINATION RISK ASSESSMENT WATER FOR AGROTOXICS IN THE PERIMETER IRRIGATION BETUME IN THE LOWER RIVER SAN FRANCISCO}

\footnotetext{
ABSTRACT

The growing agricultural activity in Betume irrigated area has caused concerns about pesticide contamination in irrigated areas. The problem becomes more important when contamination may affect the water for human use, as with the water catchment area of the

${ }^{1}$ Doutorando, UFS/IFS, São Cristovão - SE. e-mail: brandaobritto@hotmail.com

${ }^{2}$ Acadêmico de Eng. Agronômica, Bolsista Capes/UFS, São Cristovão - CE. e-mail: tassio_monteiro@hotmail.com

${ }^{3}$ Doutor, UFS/IFS, São Cristovão - SE. e-mail: anderovasco@ yahoo.com.br

${ }^{4}$ Pós Doutor, Professor, UFS, São Cristovão - SE. e-mail: Antenor.ufs@ gmail.com

${ }^{5}$ Pós Doutorando, Professor, UFS/FATEC Cariri, e-mail: carvalho_cmc@yahoo.com.br
} 
River Betume is a tributary of the River São Francisco, and supplies various regions of the state of Sergipe. The objective of the study was to evaluate the risk of contamination of surface and groundwater by pesticides and monitor water quality in areas of rice growing in the basin of River Betume. The monitoring was conducted bimonthly from March 2013 to December 2014 and the contamination risk analysis was performed using criteria of Environmental Protection Agency (EPA), GUS index and GOSS method. The results showed the presence of the following active ingredients: chlorpyrifos, tebuconazole and tetraconazole, where the resolution CONAMA 357/05 does not include the tolerance limits of pesticides in water for environmental protection for these products. When compared with the European Community standards, the concentrations of tebuconazole and chlorpyrifos were above the tolerance limit. The presence of pesticides in the region shows the population of the risks of contamination, so preventive measures in handling and application of pesticides must be taken to ensure water quality.

Keyword: environmental monitoring, contamination of surface and ground water and rice growing.

\section{INTRODUÇÃO}

O aumento da atividade agrícola desenvolvida em áreas de mananciais tem causado preocupação quanto ao potencial de contaminação, decorrentes das aplicações de agrotóxicos (Grützmacher et al., 2008). As ações antrópicas e a falta de proteção desses mananciais podem implicar em sérios problemas relacionados à potabilidade, aumentando os riscos sanitários e inviabilizando o emprego de técnicas de tratamento mais simples e menos onerosas.

Uma maior demanda de uso dos agrotóxicos está relacionado com o aumento da produção agrícola, no entanto os efeitos podem ocasionar riscos à saúde dos consumidores e manipuladores, como também ao meio ambiente (Rambow et al., 2014). Um dos maiores problemas relacionados com o uso de agrotóxico é que a maior parte aplicada não atinge os organismos, sendo assim, carreada pelas águas das chuvas, percolando ou volatilizando, representando uma ameaça aos ecossistemas como um todo (Mansano et al., 2013).

Sendo assim, a agricultura pode estar contribuindo para a contaminação dos recursos hídricos, que ocorre de maneira pontual por fontes de poluição concentrada no espaço, ou difusa que se dá por via escoamento superficial ou percolação Como exemplo, tem-se a cultura do arroz irrigado que durante o seu manejo o uso de agrotóxico pode contaminar o meio am- biente através da lixiviação ou pelo escoamento superficial (Santos et al., 2014).

As normas vigentes no país (CONAMA 357/05 e Portaria MS N ${ }^{\circ}$ 2914/11), na avaliação da qualidade da água, utilizam parâmetros de quantificação de algumas das suas propriedades e comparam com valores limite em função dos usos múltiplos. Dentre elas podemos citar o abastecimento público, proteção de comunidades aquáticas, recreação e irrigação. Com isso, há necessidade de efetivar o gerenciamento dos recursos hídricos, visando estimular a avaliação e o monitoramento da qualidade da água, em relação aos seus padrões físicos, químicos e biológicos (Knapik, 2009).

Dentro desse contexto, o monitoramento da qualidade da água torna-se um dos principais instrumentos de sustentação de uma política de planejamento e gestão de recursos hídricos, visto que funciona como um sensor que possibilita o acompanhamento do processo de uso dos cursos hídricos, apresentando seus efeitos sobre as características qualitativas das águas, visando subsidiar as ações de controle ambiental (Leli et al., 2010).

A determinação da probabilidade dos agrotóxicos de atingirem às águas subterrâneas e superficiais, é normalmente efetuada com base em índices de particionamento, considerando as propriedades físico-químicas e persistência no ambiente, como Groundwater Ubiquity Score (Gustafon, 1989), os critérios da Environmental 

IRRIGADO BETUME NO BAIXO RIO SÃO FRANCISCO

Protection Agency (Cohen et al., 1995) e o método GOSS (1992).(Sanches et al., 2003).

Diante deste contexto, o objetivo desse estudo consiste em avaliar o risco de contaminação das águas superficiais e subterrâneas e monitorar a qualidade da água na bacia hidrográfica do rio Betume no que tange o uso dos agrotóxicos na rizicultura.

\section{MATERIAL E MÉTODOS}

O perímetro irrigado Betume iniciou suas atividades em 1978 e está localizado entre os municípios de Neópolis, Ilha das Flores e Pacatuba no Baixo São Francisco nas coordenadas 24L 765.470; 8.850.015 (UTM). A administração atualmente é feita pela Companhia de Desenvolvimento dos Vales do São Francisco e do Parnaíba (CODEVASF) e contempla, especificadamente, à agricultura Familiar (Gois et al.,1992).

Está região é caracterizada pela ocorrência de várzeas inundáveis nas margens do Rio do São Francisco, com solos hidromórficos e aluviais nas partes mais baixas do relevo. Estes solos estão em áreas de topografia plana, são muito argilosos de boa fertilidade, moderadamente ácidos e apresentam baixa taxa de infiltração. Nas áreas pouco mais altas, de relevo plano, fora das várzeas, mas ainda no domínio do perímetro predominam solos arenosos (neossolos quartzarênicos) (Vargas, 1999).

A temperatura média anual variam de 22 ${ }^{\circ} \mathrm{C}$ a $28{ }^{\circ} \mathrm{C}$, com umidade relativa de $76 \%$ e uma insolação de $2.700 \mathrm{~h} \mathrm{ano}^{-1}$ (Barros e Castro, 2000). No perímetro irrigado Betume (Figura 1), no município de Neópolis - SE, há cerca de 450 pequenos produtores que cultivaram 1.392 ha de arroz no ano de 2014. Além disso, a região tem o índice pluviométrico superior a $840 \mathrm{~mm}$ por ano (Sergipe, 2012).
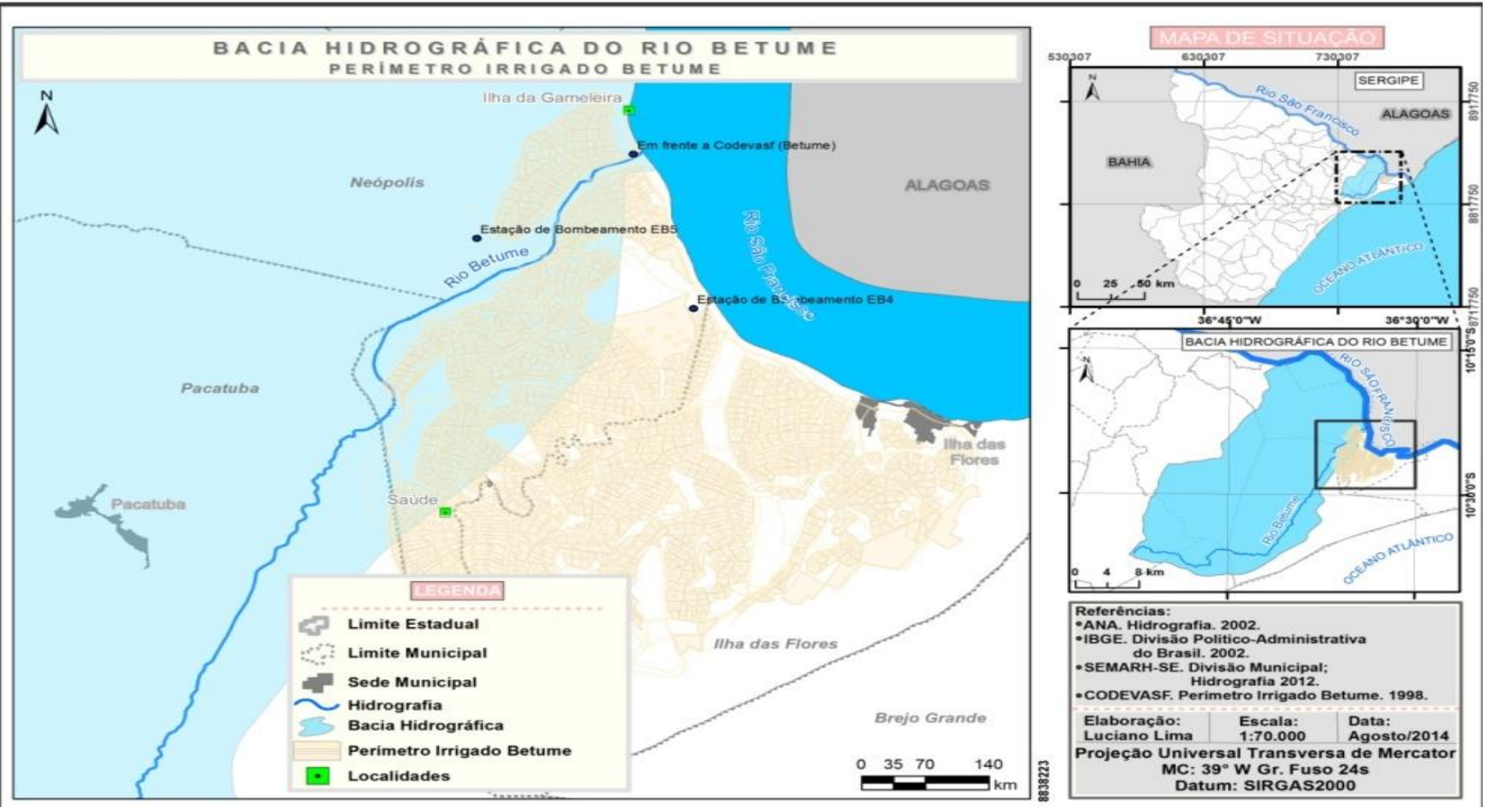

Figura 1. Localização do Perímetro Irrigado Betume, Sergipe.Fonte: ANA, 2002

Avaliação dos riscos de contaminação dos agrotóxicos nas águas subterrâneas e superficiais

A primeira etapa da pesquisa foi identificar os principais agrotóxicos utilizados na região do perímetro irrigado Betume. Foram realizadas 15 entrevistas com os irrigantes e produtores rurais, como também em pontos de venda de agrotóxicos na região de Aracaju, Propriá e Neópolis. Nestas entrevistas foram 
anotados os principais agrotóxicos comercializados para rizicultura. Estas informações foram utilizadas como base para identificar as características e propriedades físico-químicas dos agrotóxicos por meio de pesquisa na Agência Nacional de Vigilância Sanitária ANVISA e no banco de dados Pesticide Properties Database - PPD (Extoxnet, 2010).

Para a análise de riscos utilizou-se o índice de Groundwater Ubiquity Score (Gustafon, 1989), os critérios da Environmental Protection Agency (Cohen et al., 1995) e o método GOSS (1992). Esses métodos permitem avaliar a capacidade de provável risco de contaminação das águas subterrâneas e superficiais, por meio de informações sobre os princípios ativos.

O índice Groundwater Ubiquity Score GUS avalia o potencial de contaminação de água subterrânea por agrotóxico segundo a Equação 1.

$$
\text { GUS }=\log \left(t^{\frac{1}{2}} \text { Solo }\right) x(4-\log (\text { Koc }))
$$

onde:

$\mathrm{t}^{1} 1 / 2$ solo = meia vida do produto no solo (dias); Koc $=$ coeficiente de adsorção ao carbono

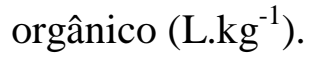

Após a obtenção do valor do índice de GUS, o princípio ativo (p.a.) é classificado em uma das categorias, definidas por faixas préestabelecidas, conforme os seguintes intervalos:
a) GUS $\leq 1,8$ = Não sofre lixiviação;
b) 1,8< GUS < 2,8 = Faixa de Transição;
c) GUS $\geq 2,8=$ Provável Lixiviação.

Outra forma utilizada para avaliar a capacidade de transporte de agrotóxico, é o critério da EPA (Cohen et al., 1995). Os princípios ativos que obedecerem às condições abaixo oferecem maior potencial de risco de transporte e, consequente tendência à contaminação, principalmente de águas subterrâneas:
a) solubilidade em água $>30 \mathrm{mg} \mathrm{L}^{-1}$;
b) coeficiente de adsorção à matéria orgânica (Koc) 300 a $500 \mathrm{~mL} \mathrm{~g}^{-1}$;
c) constante de Henry $(\mathrm{kH})<10^{-2} \mathrm{~Pa} \mathrm{~m}^{3} \mathrm{~mol}^{-1}$;
d) meia vida no solo $\left(t^{1 / 2}\right.$ solo $)>14$ a 21 dias;
e) meia vida na água ( $\mathrm{t}^{1} / 2$ água) $>175$ dias.

O método proposto por GOSS (1992), utiliza um conjunto de cláusulas e regras, apresentadas em intervalos matemáticos, pelos quais se faz a avaliação do potencial de transporte de agrotóxico associado ao sedimento ou dissolvido em água superficial (Tabela 1). As substâncias que não se enquadram em nenhum dos critérios acima são consideradas como tendo potencial médio para contaminarem águas superficiais.

Tabela 1. Método proposto por GOSS.

A) Potencial de transporte associado ao sedimento

\begin{tabular}{cccc}
\hline & $\begin{array}{c}\mathrm{t}^{1} \text { solo }_{\text {solo }} \\
(\mathrm{d})\end{array}$ & $\begin{array}{c}\mathrm{Koc} \\
\left(\mathrm{mL} \mathrm{g}^{-1}\right)\end{array}$ & $\begin{array}{c}\text { Ws } \\
\left(\mathrm{mg} \mathrm{L}^{-1}\right)\end{array}$ \\
\hline $\begin{array}{c}\text { Alto } \\
\text { Potencial }\end{array}$ & $\geq 40$ & $\geq 1000$ & - \\
& $\geq 40$ & $\geq 500$ & $\leq 0,5$ \\
\hline Baixo & $<1$ & - & - \\
Potencial & $\leq 2$ & $\leq 500$ & - \\
& $\leq 4$ & $\leq 900$ & $\geq 0,5$ \\
& $\leq 40$ & $\leq 500$ & $\geq 0,5$ \\
& $\leq 40$ & $\leq 900$ & $\geq 2$ \\
\hline
\end{tabular}

B) Potencial de transporte dissolvido em água

\begin{tabular}{cccc}
\hline & $\begin{array}{c}\mathrm{t}^{1 / 2} \text { solo } \\
(\mathrm{d})\end{array}$ & $\begin{array}{c}\text { Koc } \\
\left(\mathrm{mL} \mathrm{g}^{-1}\right)\end{array}$ & $\begin{array}{c}\text { Ws } \\
\left(\mathrm{mg} \mathrm{L}^{-1}\right)\end{array}$ \\
\hline $\begin{array}{c}\text { Alto } \\
\text { Potencial }\end{array}$ & $>35$ & $<100000$ & $\geq 1$ \\
\hline & $<35$ & $\leq 700$ & $\geq 10 \mathrm{e} \leq 100$ \\
$\begin{array}{c}\text { Baixo } \\
\text { Potencial }\end{array}$ & -1 & $\geq 100000$ & - \\
& $<35$ & - & - \\
\hline
\end{tabular}

t¹/2: meia-vida no solo (dias); Koc: coeficiente de absorção de matéria orgânica $\left(\mathrm{mL} \mathrm{g}^{-1}\right)$; Ws: solubilidade em água $(\mathrm{mg}$ $\mathrm{L}^{-1}$ ). Fonte: Milhome et al. (2009).

Segundo Pessoa et al.(2007) para calcular os valores do índice de GUS, critérios EPA e o método de GOSS foi utilizado o programa AGROSCRE da Embrapa, que faz a avaliação de tendências de transporte de princípios ativos de agrotóxico mediante o fornecimento dos dados físico-químicos de cada princípio ativo (Britto et al., 2012).

Calculados os riscos de contaminação dos princípios ativos quanto a contaminação das águas superficiais e subterrâneas foi definido 1(um) ponto de monitoramento a jusante das plantações de arroz para coleta de água na bacia 
hidrográfica do rio Betume, visando identificar resquícios de contaminações provocadas pelos agrotóxicos.

O ponto de coleta localizou-se próximo a Estação de Bombeamento EB5, coordenadas 24L 763.227; 8.848.837 (UTM). O período de coleta de amostragem de água foi de março de 2013 a dezembro de 2014, realizados por meio de coleta bimensal, totalizando 12 amostras, na camada superficial do rio em estudo. Os recipientes utilizados para coleta das amostras foram de vidro âmbar, previamente lavados e descontaminados, com papel alumínio na boca do recipiente antes da tampa com rosca e armazenado em caixa de isopor de $50 \mathrm{~L}$ com bastante gelo para o transporte. A partir das análises realizadas foi possível identificar a qualidade da água em seus respectivos períodos do ano seco e chuvoso. As amostras de água foram acondicionadas e enviadas ao laboratório sendo analisadas pelo método Multiresíduos utilizando extração líquido-líquido, a quantificação foi realizada no cromatógrafo a gás, com detectores de captura de elétrons e cromatógrafo líquido (Queiroz et al.,2003; Silvério et al., 2012; Peixe, et al., 2006).

\section{RESULTADOS E DISCUSSÃO}

Foram identificados 19 princípios ativos comumente utilizados formulados isoladamente ou em misturas, compreendendo 19 marcas comerciais, distribuídos ao longo de 17 grupos químicos diferentes (Tabela 2). Desde à criação do perímetro irrigado Betume, vem-se realizando frequentemente práticas agrícolas entre as quais, a aplicação dos agrotóxicos, que tem como objetivo manter a produtividade da rizicultura irrigada.

Tabela 2. Propriedades físico-químicas dos princípios ativos dos agrotóxicos, a $20-25{ }^{\circ} \mathrm{C}$, usados na região da bacia hidrográfica do rio Betume.

\begin{tabular}{|c|c|c|c|c|c|c|c|c|c|}
\hline Princípio Ativo* & Grupo Quimico & $\mathrm{Uso}^{\mathrm{a}}$ & $\begin{array}{c}\mathrm{T}^{1 / 2} \\
\text { Solo }^{\mathrm{b}}\end{array}$ & $\begin{array}{l}\mathrm{T}^{1 / 2} \\
\text { Água }^{\mathrm{c}}\end{array}$ & $\operatorname{Koc}^{d}$ & $\mathrm{Ws}^{\mathrm{e}}$ & $V p^{f}$ & $\mathrm{KH}^{\mathrm{g}}$ & $\begin{array}{c}\text { Clas } \\
\text { Toxic }^{\text {h }} \\
\end{array}$ \\
\hline $1-2,4-\mathrm{D}$ & Ácido Ariloxialcanóico & $\mathrm{O}$ & 34 & 39 & 46 & 27600 & $1,8 \mathrm{E}-07$ & 0,011 & I \\
\hline 2-Bispiribaque-sódico & $\begin{array}{l}\text { Ácido } \\
\text { Pirimidiniloxibenzóico }\end{array}$ & $\mathrm{O}$ & 138 & 3 & $2,09 \mathrm{E}-03$ & 73300 & $3,00 \mathrm{E}-11$ & $5,05 \mathrm{E}-09$ & II \\
\hline 3-Cipermetrina & Piretroide & $\mathrm{O}$ & 60 & 179 & 82900 & 0,01 & 0,02 & $2,30 \mathrm{E}-04$ & II \\
\hline 4-Clorpirifós & Organofosforados & $\mathrm{R} / \mathrm{O}$ & 50 & 25,5 & 8151 & 1,05 & $4,78 \mathrm{E}-01$ & $2,40 \mathrm{E}-05$ & II \\
\hline 5-Deltametrina & Piretroide & $\mathrm{O}$ & 23 & 92 & 460.000 & 2,00E-06 & $3,13 \mathrm{E}-02$ & $1,24 \mathrm{E}-02$ & III \\
\hline 6-Epoxiconazol & Triazol & $\mathrm{O}$ & 354 & 350 & 1073 & 7,1 & $4,71 \mathrm{E}-04$ & $1 \mathrm{E}-07$ & III \\
\hline 7-Imazapique & Imidazolinona & $\mathrm{R} / \mathrm{O}$ & 140 & 30 & 206 & 36000 & 0,3 & $2,50 \mathrm{E}-03$ & II \\
\hline 8-Imazatapir & Imidazolinona & $\mathrm{R} / \mathrm{O}$ & 90 & 3 & 100 & 13000 & $1,30 \mathrm{E}-03$ & $1,20 \mathrm{E}-07$ & III \\
\hline 9-Ivermectina & Organofosforados & $\mathrm{O}$ & 36 & 45 & 14100 & $7,50 \mathrm{E}-03$ & 4,1E-08 & $1,10 \mathrm{E}-11$ & I \\
\hline 11-Mancozebe & Ditiocarbamato & $\mathrm{O}$ & 70 & 13 & 2000 & 6,2 & $5,90 \mathrm{E}-04$ & $1,00 \mathrm{E}-05$ & III \\
\hline 12-Piraclostrobina & Estrobilurina & $\mathrm{O}$ & 32 & 660 & 11000 & 1,9 & $5,31 \mathrm{E}-06$ & $2,60 \mathrm{E}-05$ & II \\
\hline 13-Pirazossulfurom-étilico & Sulfoniluréia & $\mathrm{R} / \mathrm{O}$ & 28 & 15 & 250 & 9,8 & $1,00 \mathrm{E}-02$ & $4,00 \mathrm{E}-05$ & III \\
\hline 14-Tebuconazol & Triazol & $\mathrm{O}$ & 62 & 42 & 769 & 36 & $1,00 \mathrm{E}-05$ & $1,07 \mathrm{E}-06$ & III \\
\hline 15-Tetraconazol & Triazol & $\mathrm{O}$ & 403 & - & 3,2838 & 1500 & - & $1,80 \mathrm{E}-01$ & II \\
\hline 16-Tiametoxam & Neonecotinoide & $\mathrm{R} / \mathrm{O}$ & 50 & 180 & 56,2 & 4100 & $4,70 \mathrm{E}-10$ & $6,6 \mathrm{E}-06$ & III \\
\hline 17-Tiofanato-Métilico & Benzimidazol & $\mathrm{O}$ & 10 & 36 & 1830 & 3,5 & 0,000081 & $7,12 \mathrm{E}-08$ & III \\
\hline 18-Triciclazol & Benzotiazol & $\mathrm{O}$ & 21 & 92 & 169 & 0,536 & $5,86 \mathrm{E}-07$ & $1,00 \mathrm{E}-07$ & II \\
\hline 19-Trifloxistrobina & Estrobilurina & $\mathrm{O}$ & 7 & 40 & 2377 & 0,61 & $2,30 \mathrm{E}-03$ & 0,024 & III \\
\hline
\end{tabular}

Nomenclatura de acordo com as regras brasileiras*; "_" valor não encontrado na literatura ou não calculado por falta de parâmetros; a = usos na cultura da cana-de-açúcar: R - Pré-emergente; O - Pós-emergente; $\mathrm{M}$ - Maturador; $\mathrm{b}=$ meia-vida no solo, em dias; $\mathrm{c}=$ meia-vida na água, em dias; $\mathrm{d}$ = coeficiente de adsorção normalizado pela fração de carbono orgânico do solo $\left(\mathrm{L} \mathrm{kg}^{-1}\right)$; e = solubilidade em água (mg L ${ }^{1}$ ); f = pressão de vapor, em MPa., g = constante de Henry kH.; h = classe toxicológica (I - extremamente tóxico; II - altamente tóxico; III medianamente tóxico; IV - pouco tóxico). Dados extraídos de: (PPDB, 2010 e Extoxnet, 2010). 
Na região do perímetro irrigado Betume, as principais atividades é a rizicultura e a criação de gado de corte. Durante a entressafra da cultura do arroz alguns agricultores utilizam restos da palhada da cultura do arroz como pastejo. Por conta das práticas realizadas na região, é encontrada facilmente a utilização de agrotóxicos dos grupos químicos piretroide, imidazolinona, triazol e estroubirulina sendo todos eles registrados para a cultura do arroz ou para o controle de exoparasitas "carrapato". Todos os grupos de princípios ativos estão registrados junto ao Ministério da Agricultura, Pecuária e Abastecimento e na Secretaria de Agricultura e Abastecimento do Estado de Sergipe.

Segundo Silva et al. (2007), a persistência de um determinado composto no ambiente é determinado geralmente pela meia-vida, que significa o tempo necessário para que ocorra a dissipação de pelo menos $50 \%$ da quantidade de agrotóxico aplicada inicialmente. Alguns princípios ativos apresentaram elevados valores de persistência na água como por exemplo: cipermetrina, epoxiconazol, piraclostrobina e tiametoxam (Tabela 2). Estes princípios ativos apresentaram alta persistência no solo ou na água, aumentando os possíveis danos ao meio ambiente, pois os mesmos têm potencial para uma possível contaminação de águas subterrâneas, como em casos de lençóis freáticos não confinados.

$\mathrm{Na}$ Tabela 3 é possível observar os resultados das análises de potencial de contaminação da água subterrâneas pelos princípios ativos mais utilizados na produção de arroz na região do perímetro irrigado Betume, conforme os critérios "screening", sugeridos pelo EPA e pelo índice GUS. Perante o índice GUS os princípios ativos 2,4-D, bispiribaquesódico, imazapique, imazatapir, lambdacialotrina, tetraconazol e tiametoxam apresentaram possíveis contaminações das águas subterrâneas. Por outro lado alguns princípios ativos encontraram-se em zona de transição como por exemplo: epoxiconazol, pirazossulfurom-étilico, tebuconazol e triciclazol. Já os demais princípios ativos quando analisados pelo EPA mostraram tendência à contaminação.

Tabela 3. Avaliação de risco de contaminação de águas subterrâneas, pelo índice de GUS, e critérios da EPA.

\begin{tabular}{llccc}
\hline \multicolumn{1}{c}{ Princípio Ativo* } & \multicolumn{1}{c}{ Grupo Químico } & GUS & EPA \\
\hline 1-2,4-D & Ácido Ariloxialcanóico & $\mathrm{L}$ & 3,579437 & $\mathrm{~L}$ \\
2-Bispiribaque-sódico & Ácido Pirimidiniloxibenzóico & $\mathrm{L}$ & 14,29408 & $\mathrm{~L}$ \\
3-Cipermetrina & Piretroide & $\mathrm{NL}$ & $-1,633329$ & $\mathrm{~L}$ \\
4-Clorpirifós & Organofosforados & $\mathrm{NL}$ & 0,150850 & $\mathrm{~L}$ \\
5-Deltametrina & Piretroide & $\mathrm{NL}$ & $-2,264224$ & $\mathrm{~L}$ \\
6-Epoxiconazol & Triazol & $\mathrm{T}$ & 2,471005 & $\mathrm{~L}$ \\
7-Imazapique & Imidazolinona & $\mathrm{L}$ & 3,618657 & $\mathrm{~L}$ \\
8-Imazatapir & Imidazolinona & $\mathrm{L}$ & 3,908485 & $\mathrm{~L}$ \\
9-Ivermectina & Organofosforados & $\mathrm{L}$ & $-0,232230$ & $\mathrm{~L}$ \\
10-Lambda-cialotrina & Piretroide & $\mathrm{NL}$ & 1,2832845 & $\mathrm{~L}$ \\
11-Mancozebe & Ditiocarbamato & $\mathrm{NL}$ & $-6,23 \mathrm{E}-02$ & $\mathrm{~L}$ \\
12-Piraclostrobina & Estrobilurina & $\mathrm{T}$ & 2,318434 & $\mathrm{~L}$ \\
13-Pirazossulfurom-étilico & Sulfoniluréia & $\mathrm{T}$ & 1,996857 & $\mathrm{~L}$ \\
14-Tebuconazol & Triazol & $\mathrm{L}$ & 9,075902 & $\mathrm{~L}$ \\
15-Tetraconazol & Triazol & $\mathrm{L}$ & 3,823131 & $\mathrm{~L}$ \\
16-Tiametoxam & Neonecotinoide & $\mathrm{NL}$ & 0,737549 & $\mathrm{~L}$ \\
17-Tiofanato-Métilico & Benzimidazol & $\mathrm{T}$ & 2,343123 & $\mathrm{~L}$ \\
18-Triciclazol & Benzotiazol & $\mathrm{NL}$ & 0,527317 & $\mathrm{~L}$ \\
19-Trifloxistrobina & Estrobilurina & $\mathrm{n}$ &
\end{tabular}

Resultados fornecidos pelo programa AGROSCRE, GUS = Índice do potencial de lixiviação, onde L= Provável lixiviação; $\mathrm{NL}=$ Não lixívia; $\mathrm{T}$ = Faixa de transição; $\mathrm{EPA}=$ avaliação pelo critério da EPA (onde NA= Não avaliado por falta de informações; $\mathrm{L}=$ Provável lixiviação, $\mathrm{NL}$ = Não sofre lixiviação). 
Comparando os dados da Tabela 2 e Tabela 3, pelo método GUS, os dados de meiavida do solo $\left(\mathrm{T}^{1 / 2}\right.$ solo) e de coeficiente de adsorção ao carbono orgânico (Koc), referente ao 2,4-D, bispiribaque-sódico, imazapique, imazatapir, lambda-cialotrina, tiametoxam e o tetraconazol apresentaram tendência à lixiviação para águas subterrânea. Observando os métodos utilizados no presente trabalho foi possível notar certas características de análise de cada método, ou seja, ao comparar o GUS com o EPA, notase que quanto menor o valor do Koc o princípio ativo sofrerá provável lixiviação, pois o mesmo terá baixa capacidade de ser adsorvido pela matéria orgânica existente no solo e consequentemente sofrerá lixiviação e/ou percolação, e assim contaminando os recursos hídricos existentes.

De acordo com o índice GUS os resultados mostrados na Figura 2 demonstram que dos 19 princípios ativos mais utilizados e calculados pelo método GUS, 7 princípios ativos apresentaram valores que indicam um provável risco de lixiviação (GUS $\geq 2,8$ ), sendo que o lambda-cialotrina, tetraconazol e bispiribaque-sódico apresentaram os maiores valores para o índice GUS, de 6,63, 9,07 e 14,29 respectivamente.

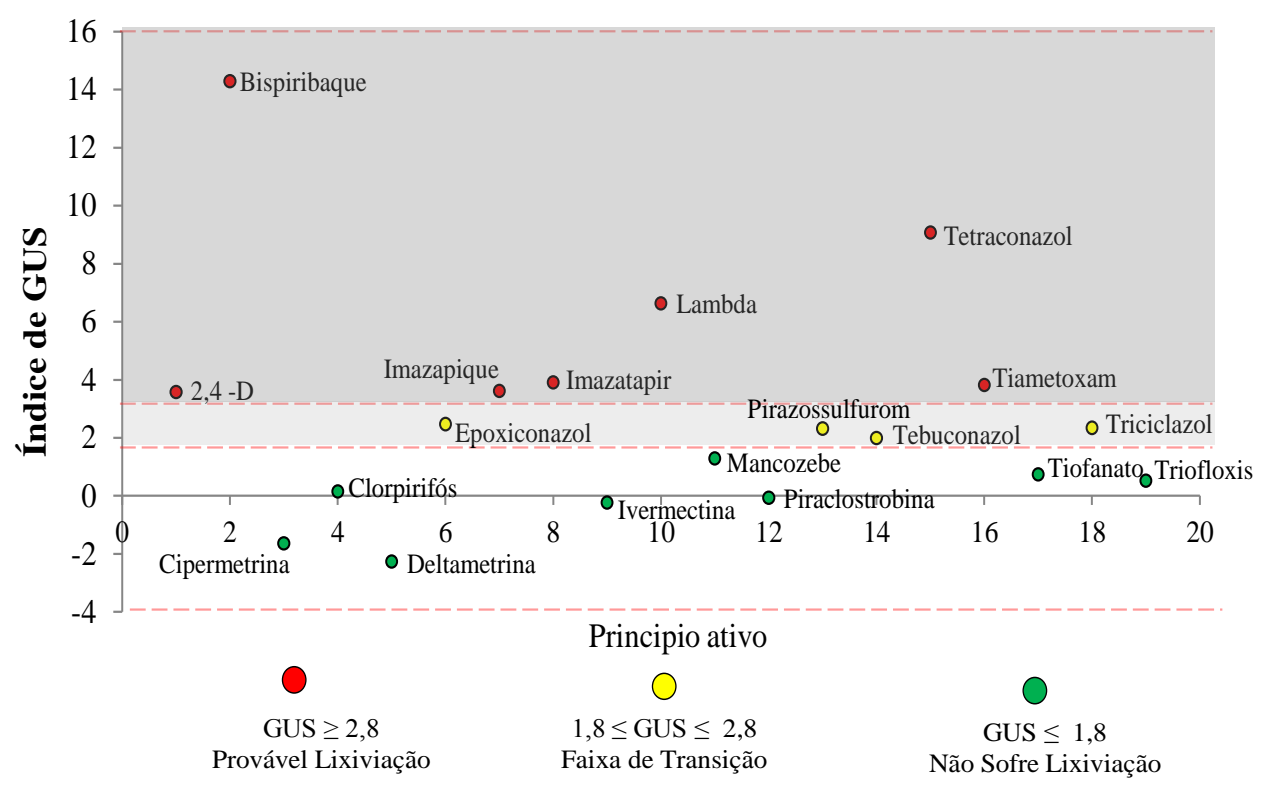

Figura 2. Resultados da avaliação de risco de contaminação de águas subterrâneas com base no índice de GUS, na bacia hidrográfica do rio Betume.

Estes valores indicam uma grande possibilidade destas moléculas lixiviarem e assim, contaminar os rios, lagos e águas subterrâneas da região onde foram aplicados. Resultados semelhantes foram encontrados na bacia do Pantanal Mato-Grossense e em lavouras de arroz irrigado na Lagoa Mirim no Rio Grande do Sul (Dores e Calheiros, 2008; Gomes e Barizon, 2014).

Oito princípios ativos não sofrem lixiviação (GUS $\leq 1,8)$ e nem mesmo percolação nos perfis do solo são eles: deltametrina, cipermetrina, piraclostrobina, clorpirifós, trifloxistrobina, tiofanato-métilico, mancozebe e ivermectina. Já o tebuconazol, pirazossulfurom-étilico, epoxiconazol e o triciclazol apresentaram valores na faixa de transição $(1,8 \leq$ GUS $\geq 2,8)$ podendo ou não lixiviar no solo (Figura 2).

Os agrotóxicos, que estão sendo evidenciados na contaminação dos rios, foram divididos em duas classes: aqueles que podem ser transportado através da sua dissolução em água e aqueles que são transportados associados 
aos sedimentos em suspensão existentes no corpo hídrico, de acordo com o método GOSS.

Dentre os princípios ativos com alto potencial de transporte dissolvidos em água estão: bispiribaque-sódico, clorpirifós, epoxiconazol, imazapique, imazatapir, mancozebe, tebuconazol, tetraconazol e tiametoxam. Sendo os agrotóxicos que apresentam maior capacidade de transporte sedimentos são eles: cipermetrina, clorpirifós, epoxiconazol e mancozebe (Tabela 4).

Tabela 4. Classificação dos princípios ativos pelo método GOSS obtido com o programa AGROSCRE na bacia do hidrográfica do rio Betume.

\begin{tabular}{|c|c|c|c|}
\hline \multirow{2}{*}{ Princípio Ativo* } & \multirow{2}{*}{ Grupo Químico } & \multicolumn{2}{|c|}{ GOSS } \\
\hline & & Sedimento & Dissolvido \\
\hline $1-2,4-\mathrm{D}$ & Ácido Ariloxialcanóico & B & M \\
\hline 2-Bispiribaque-sódico & Ácido Pirimidiniloxibenzóico & M & A \\
\hline 3-Cipermetrina & Piretroide & A & M \\
\hline 4-Clorpirifós & Organofosforados & A & A \\
\hline 5-Deltametrina & Piretroide & M & $\mathrm{B}$ \\
\hline 6-Epoxiconazol & Triazol & A & A \\
\hline 7-Imazapique & Imidazolinona & M & A \\
\hline 8-Imazatapir & Imidazolinona & M & A \\
\hline 9-Ivermectina & Organofosforados & M & M \\
\hline 10-Lambda-cialotrina & Piretroide & M & $\mathrm{B}$ \\
\hline 11-Mancozebe & Ditiocarbamato & A & A \\
\hline 12-Piraclostrobina & Estrobilurina & M & M \\
\hline 13-Pirazossulfurom-étilico & Sulfoniluréia & $\mathrm{B}$ & M \\
\hline 14-Tebuconazol & Triazol & M & A \\
\hline 15-Tetraconazol & Triazol & M & A \\
\hline 16-Tiametoxam & Neonecotinoide & M & A \\
\hline 17-Tiofanato-Métilico & Benzimidazol & M & M \\
\hline 18-Triciclazol & Benzotiazol & $\mathrm{B}$ & M \\
\hline 19-Trifloxistrobina & Estrobilurina & M & M \\
\hline
\end{tabular}

A - Alto potencial de transporte; B - baixo potencial de transporte; M - médio potencial de transporte e NA - não analisado

Nas coletas de água bimensais, realizadas durante o período de março de 2013 a dezembro de 2014, foram detectados três princípios ativos utilizados nas práticas agrícolas da região do perímetro irrigado Betume são eles: clorpirifós, tebuconazol e o tetrabuconazol. A presença destes agrotóxicos na bacia hidrográfica do rio Betume foi detectada nos meses de novembro de 2013, março, outubro e dezembro de 2014. Não foram encontradas concentrações superiores ao Limite de Quantificação (LQ): 0,03 $\mu \mathrm{g} . \mathrm{L}^{-1}$ nas demais amostras realizadas (Tabela 5). 
Tabela 5. Concentração do Clorpirifós, Tebuconazole e Tetraconozole no período chuvoso e seco.

\begin{tabular}{|c|c|c|c|c|c|}
\hline \multirow[b]{2}{*}{$\begin{array}{l}\text { Data da } \\
\text { coleta }\end{array}$} & \multirow[b]{2}{*}{$\begin{array}{l}\text { Precipitação pluvial } \\
\text { (mm) }\end{array}$} & \multirow[b]{2}{*}{ Período } & \multicolumn{3}{|c|}{ Rio Betume } \\
\hline & & & $\begin{array}{c}\text { Clorpirifós } \\
\left(\mu \mathrm{g} . \mathrm{L}^{-1}\right)\end{array}$ & $\begin{array}{l}\text { Tebuconazole } \\
\left(\mu \mathrm{g} . \mathrm{L}^{-1}\right)\end{array}$ & $\begin{array}{l}\text { Tetraconazole } \\
\left(\mu \mathrm{g} . \mathrm{L}^{-1}\right)\end{array}$ \\
\hline $14 / 03 / 2013$ & 37,25 & seco & $<\mathrm{LD}$ & $<\mathrm{LD}$ & $<\mathrm{LD}$ \\
\hline $27 / 05 / 2013$ & 200,5 & chuvoso & $<\mathrm{LD}$ & $<\mathrm{LD}$ & $<\mathrm{LD}$ \\
\hline $25 / 07 / 2013$ & 202,9 & chuvoso & $<\mathrm{LD}$ & $<\mathrm{LD}$ & $<\mathrm{LD}$ \\
\hline $23 / 09 / 2013$ & 48,3 & seco & $<\mathrm{LD}$ & $<\mathrm{LD}$ & $<\mathrm{LD}$ \\
\hline $20 / 11 / 2013$ & 93,75 & seco & $<\mathrm{LD}$ & 0,48 & $<\mathrm{LD}$ \\
\hline 28/01/2014 & 9,75 & seco & $<\mathrm{LD}$ & $<\mathrm{LD}$ & $<\mathrm{LD}$ \\
\hline $17 / 03 / 2014$ & 73 & seco & 0,12 & $<\mathrm{LD}$ & $<\mathrm{LD}$ \\
\hline $14 / 05 / 2014$ & 253,5 & chuvoso & $<\mathrm{LD}$ & $<\mathrm{LD}$ & $<\mathrm{LD}$ \\
\hline $15 / 07 / 2014$ & 238,25 & chuvoso & $<\mathrm{LD}$ & $<\mathrm{LD}$ & $<\mathrm{LD}$ \\
\hline 20/08/2014 & 102,5 & chuvoso & $<\mathrm{LD}$ & $<\mathrm{LD}$ & $<\mathrm{LD}$ \\
\hline $14 / 10 / 2014$ & 202,75 & seco & $<\mathrm{LD}$ & 0,08 & $<\mathrm{LD}$ \\
\hline $03 / 12 / 2014$ & 18,25 & seco & $<\mathrm{LD}$ & 0,09 & 0,08 \\
\hline
\end{tabular}

Nota: < LD - Valor abaixo do Limite de Detecção do método.

Os valores encontrados para clorpirifós em 03/2013 com concentrações de $0,12 \mu \mathrm{g}$ $\mathrm{L}^{-1}$, tebuconazole nos meses $11 / 2013 \mathrm{com}$ $0,48 \mu \mathrm{g} \mathrm{L}^{-1}, 10 / 2014$ com $0,08 \mu \mathrm{g} \mathrm{L}^{-1}$ e $12 / 2014$ com $0,09 \mu \mathrm{g} \mathrm{L}^{-1}$, para o tetraconazole em 12/2014 com 0,09 $\mu \mathrm{g} \mathrm{L}^{-1}$ (Figura 3). No Brasil, a Resolução CONAMA no 357/05 que estabelece os limites máximos de contaminantes em águas, não contemplam a maioria dos pesticidas em uso atualmente como o clorpirifós, tebuconazol e tetraconozole.

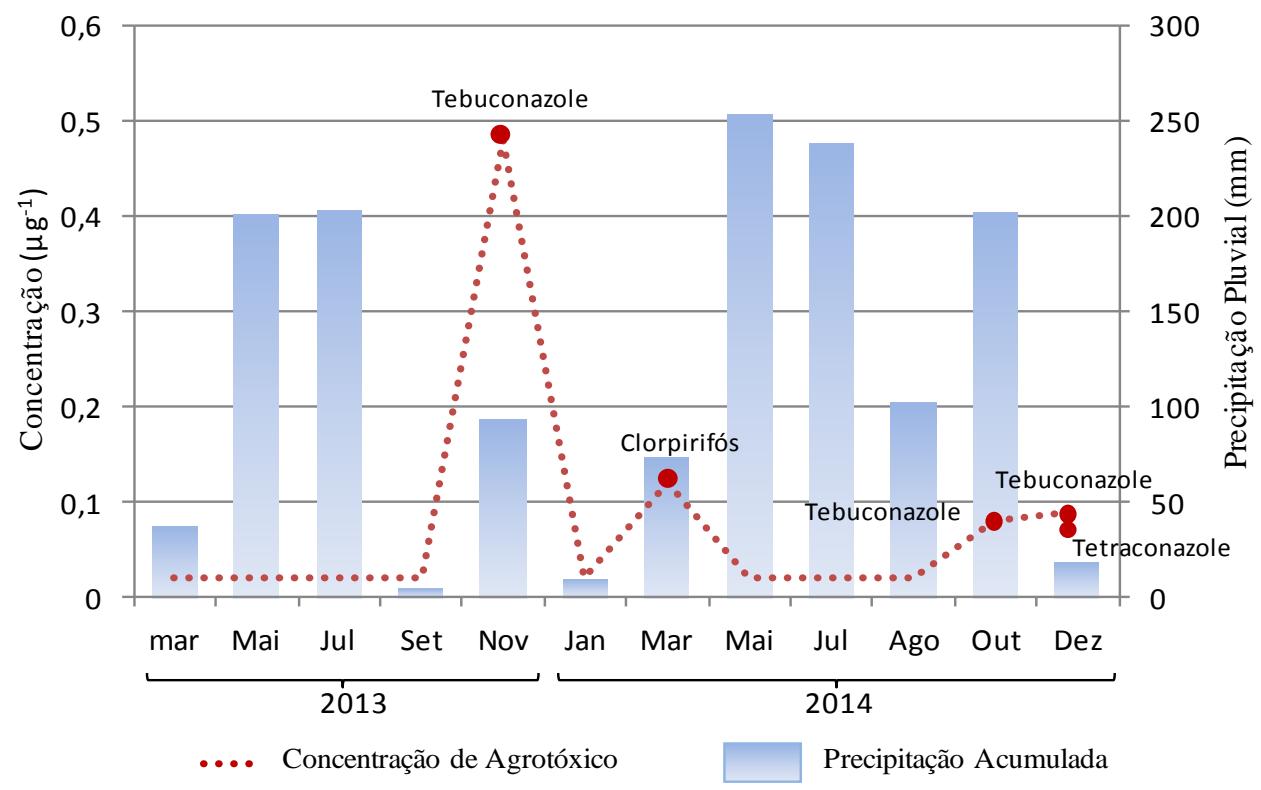

Figura 3. Comportamento da precipitação pluvial ocorrida no rio Betume de 03/2013 a 12/2014, para estação de Neópolis, e a concentração dos p.a. clorpirifós, tebuconazol e tetraconozole.

Para a Portaria MS No 2914/2011 o valor máximo permitido (VMP) para o clorpirifós é de $30 \mu \mathrm{g} \mathrm{L}^{-1}$ e o tebuconazol é de $180 \mu \mathrm{g} \mathrm{L}^{-1}$ em água destinada ao consumo humano proveniente de sistema e solução alternativa de abastecimento de água. Comparando aos valores 
detectados no monitoramento, estes estão bem abaixo do VMP. Para o Tetraconazole não se tem limite de tolerância (BRASIL, 2011).

Quando comparados com o padrão de potabilidade da Agência de Proteção Ambiental dos Estados Unidos (EPA), tem-se os limites máximos de $28 \mu \mathrm{g} . \mathrm{L}^{-1}\left(0,028 \mathrm{mg} \cdot \mathrm{kg}^{-1}\right)$ para ingestão diária aceitável (IDA) pelo homem (Extoxnet, 2010). Os valores detectados no rio Betume estão abaixo da IDA.

A permissão de uso de agrotóxicos está condicionada há vários estudos, entre eles a toxicidade avaliada pela determinação da ingestão diária aceitável (IDA) e do limite máximo de resíduo (LMR). IDA é expressa em mg. $\mathrm{kg}^{-1} \mathrm{de}$ massa corpórea, estabelecida por longa avaliação toxicológica em animais experimentais, definida como sendo "a quantidade de uma substância química que pode ser ingerida diariamente pelo homem durante toda a vida, sem risco apreciável a sua saúde, à luz dos conhecimentos disponíveis na época da avaliação (Lima e Correa, 2015). Segundo a ANVISA o LMR do tebuconazole no feijão é de $100 \mu \mathrm{g} . \mathrm{L}^{-1}\left(0,1 \mathrm{mg} \cdot \mathrm{kg}^{-1}\right)$ portanto, os valores detectados no rio Betume estão abaixo do LMR (ANVISA,2011).

Ainda quando comparados com a Comunidade Europeia esta admite concentração máxima de $0,1 \mu \mathrm{g} \mathrm{L}^{-1}$ para qualquer agrotóxico em águas destinadas para consumo humano e em $0,5 \mu \mathrm{g} \mathrm{L}^{-1}$ para o total de resíduos, sem deixar claro se deve, ou não, considerar também produtos de transformação (Filizola et al., 2002; Armas et al., 2007). Tanto o clorpirifós, como o tebuconazole apresentaram valores acima do limite de tolerância.

Outro fato, é a presença do princípio ativo, clorpirifós, que é um carrapaticida nesta região ocorreu na entressafra do arroz (fevereiro a maio). Neste período é comum alguns os produtores deixarem o gado para se alimentar, após a colheita do grão (palhada do arroz).

No caso do tebuconazole e tetraconozole as suas concentrações foram detectadas em novembro de 2013, outubro e dezembro de
2014. Nos demais meses não foi possível detectar sua presença, pois o princípio ativo não existia na água ou estava abaixo do limite de detecção dos métodos utilizados em laboratório.

O Tebuconazole, que é um fungicida e combate as doenças Cárie-do-grão (Tilletia barclayana), Brusone (Pyricularia grisea) e a Mancha-parda (Bipolaris oryzae). Os trabalhadores iniciaram o uso deste agrotóxico, para combater a doença do Brusone. Este é o agente causal da doença, Pyriculariagrisea (Cooke) Sacc, que possui capacidade de infectar várias gramíneas dentre elas $\mathrm{o}$ arroz. A enfermidade desenvolve rapidamente quando existem condições adequadas como: períodos de orvalho e associados a chuvas leves, as quais mantêm a umidade sobre as folhas (Scheuermann e Eberhardt, 2011).

No perímetro irrigado Betume, o Bruzone foi detectado em algumas propriedades e por orientação técnica foi prescrito o uso do fungicida para combater a doença e evitar a queda da produtividade das lavouras de arroz.

\section{CONCLUSÕES}

1. As concentrações de clorpirifós, tetraconozole e tebuconazole encontrados no rio Betume apresentaram valores abaixo, quando comparados com os padrões de ingestão diária aceitável (IDA) da ANVISA e EPA. Mas, de acordo com normas da Comunidade Europeia, as concentrações de tebuconazole e clorpirifós foram elevadas nos meses de novembro de 2013 e março de 2014.

3. O clorpirifós apresentou grande potencial de transporte por sedimentos, alta capacidade de transporte na água e provável lixiviação comprometendo os recursos hídricos subterrâneos, o tetraconozole e tebuconazole apresentaram alto potencial de transporte em sedimentos, média capacidade de transporte em água e provável lixiviação desta forma comprometendo as águas subterrâneas. 
4. A continuidade no monitoramento da qualidade da água possibilitará a identificação de possíveis novos princípios ativos aplicados na rizicultura, atualmente predominante na região, e assim preservar a qualidade da água da bacia hidrográfica do rio Betume e a sustentabilidade ambiental desta região.

\section{AGRADECIMENTOS}

Os autores agradecem ao projeto Águas do São Francisco, patrocinado pela PETROBRAS por meio do programa PETROBRAS socioambiental e realizado pela Universidade Federal de Sergipe - UFS e Sergipe Parque Tecnológico - SERGIPETEC.

\section{REFERÊNCIAS BIBLIOGRÁFICAS}

ANA - Agência Nacional das Águas. A Evolução da Gestão dos Recursos Hídricos no Brasil. Brasília: ANA, 2002. 64p. Edição comemorativa do dia mundial da água.

ANVISA - Agencia Nacional de Vigilância Sanitária. Programa de Análise de Resíduos de Agrotóxicos em Alimentos. Relatório de atividade 2010. Disponível em: http://portal.anvisa.gov.br/wps/portal/anvisa/anv isa/home/agrotoxicotoxicologia - dezembro/ 2011. acesso em 21 de janeiro de 2015.

ARMAS, MONTEIRO, R. T. R.; ANTUNES, P. M.; SANTOS, M. A. P. F.; CAMARGO, P. B.; ABAKERLI, R. B., Diagnóstico espaçotemporal da ocorrência de herbicidas nas águas superficiais e sedimentos do Rio Corumbataí e principais afluentes. Química Nova, v. 30, n. 05, p. 1119-1127, 2007.

BARROS, L. C. G.; CASTRO, A. L. ,Recomendações Técnicas para a Criação de Peixes no Baixo São Francisco. EMBRAPA , ISSN 1517-1310, janeiro,2000

BRASIL. Ministério da Saúde. Portaria no 2914 de 12 de dezembro de 2011. Dispõe sobre os procedimentos e responsabilidades relativos ao controle e vigilância da qualidade da água para consumo humano e seu padrão de potabilidade. Diário Oficial da União, Brasília, DF, 2011. Disponível em: Acesso em: 21 de fevereiro de 2015.

BRITTO, F. B.; VASCO, A. N.; PEREIRA, A. P. S.; JÚNIOR, A. V. M.; NOGUEIRA, L. C. Herbicidas no alto Rio Poxim, Sergipe e os riscos de contaminação dos recursos hídricos. Revista Ciência Agronômica., v. 43, n. 2, p. 390-398, abr-jun, 2012.

COHEN, S. Z.; WAUCHOPE, R. D.; KLEIN, A. W.; EADSPORTH, C. V.; GRANCY, R. Offsite transport of pesticides in water mathematical models of pesticide leaching and runoff. Pure and Applied Chemistry, v.67, p.2109- 2148, 1995.

CONAMA - Conselho Nacional do Meio Ambiente. Resolução no 357, de 17 de março de 2005. Publicada no Diário Oficial da União $\mathrm{n}^{\circ}$ 053, de 18 de março de 2005, p 58-63. Disponível em www.mma.gov.br/port/ conama/res/res05/res35705.pdf. Acesso em 8 de dezembro de 2014.

DORES, E. F. G. C.; CALHEIROS, D. F. Contaminação por agrotóxicos na bacia do rio Miranda, Pantanal (MS). Revista Brasileira de Agroecologia, v. 3, Suplemento especial, 2008.

EXTOXNET - EXTENSION TOXICOLOGY NETWORK Pesticides active ingredient profiles. 2010. Disponível em: <http:// pmep.cce.cornell.edu/profiles/extoxnet $>$.

Acesso em: 7de janeiro de 2015.

FILIZOLA, H. F.; Ferracini, V. L., Sans, L. M. A.; Gomes M. A. F.; Ferreira, C. J. A., Monitoramento e avaliação do risco de contaminação por pesticidas em água superficial e subterrânea na região de Guaíra. Pesquisa Agropecuária Brasileira, v. 37, n. 05, p. 659-667, 2002.

GOIS, J. A.; PAIVA, M. F. A.; TAVARES, 
S.M.G. Projeto de Irrigação no Vale do do Baixo São Francisco,Instituto de Pesquisa Econômica Aplicada, Brasília,1992

GOMES, M. A. F.; BARIZON, R. R. M. Panorama da Contaminação Ambiental por Agrotóxicos e Nitrato de origem Agrícola no Brasil: Cenário 1992/2011- Embrapa Meio Ambiente, Jaguariúna, SP, 2014.

GOSS, D. W., Screening procedure for soils and pesticides for potential water quality impacts. Weed Technology, v. 06, n. 03, p. 701-708, 1992.

GRÜTZMACHER，D. D.;GRÜTZMACHERI, A. D.;AGOSTINETTO, D.; LOECK, A. E.; ROMAN, R.; PEIXOTO, S.C; ZANELLA, R., Monitoramento de agrotóxicos em dois mananciais hídricos no Sul do Brasil. Revista Brasileira de Engenharia Agrícola e Ambiental, v. 12, n. 06, p. 632-637, 2008.

GUSTAFSON, D. I. Groundwater ubiquity score: a simple method for assessing pesticide leachability. Environmental Toxicology and Chemistry, v. 08, n. 04, p. 339-357, 1989.

KNAPIK, H. G. Reflexões Sobre monitoramento, modelagem e calibração na gestão de recursos hídricos: estudo de caso da qualidade da água da Bacia do Alto Iguaçu. Dissertação apresentada ao Curso de PósGraduação em Engenharia de Recursos Hídricos e Ambiental da Universidade Federal do Paraná, Curitiba, 2009

LELI, I. T.; STEVAUX, J. C.; NÓBREGA, M. T. Produção e transporte da carga suspensa fluvial: teoria e método para rios de médio porte. Boletim de geografia, v. 28 , n. 1 , p. 43$58,2010$.

LIMA, M. A; CORREA, I. M. Entendendo os limites de resíduos de agrotóxicos em alimentos. Infobibos Informações Tecnológicas, disponível em: http://www.infobibos.com/ Artigos/2012_2/entendendoalimentos/index.htm Acesso em: 7 janeiro. 2015.
MANSANO, A. S.; MOREIRA, R. A.; ROCHA, O. Toxicidade aguda do agrotóxico carbofurano ao cladócero ceriodaphnia silvestrii daday, 1902. Fórum Ambiental da Alta Paulista, v. 9, n. 11, 2013, p. 91-103.

MILHOME, M. A. L.; SOUSA, D. O. B.; LIMA, F.A.F.; NASCIMENTO, R. F., Avaliação do potencial de contaminação de águas superficiais e subterrâneas por pesticidas aplicados na agricultura do Baixo Jaguaribe, CE. Engenharia Sanitária e Ambiental, v. 14, n. 03 p. 363-372, 2009.

PEIXE, T. S.; NASCIMENTO, E. S.; ROSA, H. V. D., Determinação de fenol urinário por cromatografia em fase gasosa em trabalhadores que utilizam resinas fenólicas em fundições, Revista Brasileira Ciências Farmacêuticas vol.42 no.2 São Paulo Apr./June 2006

PESSOA, M.C.P.Y.; CHAIM, A.; FERRACINI, V.L.; SCRAMIN, S. Manual do usuário do programa AGROSCRE: apoio à avaliação de tendências de transporte de princípios ativos de agrotóxicos, EMBRAPA MEIO AMBIENTE, Jaguariúna-SP. 2007

PPDB - THE PESTICIDE PROPERTIES DATABASE. Developed by the Agriculture \& Environment Research Unit (AERU), University of Hertfordshire, funded by UK national sources and the EU-funded FOOTPRINT project (FP6 - SSP-022704). $2010 . \quad$ Disponível em: <http://sitem.herts.ac.uk/aeru/footprint/index2.h tm>. Acesso em Acesso em: 15 jan. 2015.

QUEIROZ, S. C. N.; NOGUEIRA, R. T.; SAITO, M. L. Método para determinação multiresíduos de imazaquim, tebuconazole, fluazifop-p-butil e fluazifop-p em vagem de soja. Separata de: Congreso Virtual Iberoamericano sobre Gestión de Calidad en Laboratorios, Valladolid. Resúmenes... Valladolid: ITACYL, 2003.

RAMBOW, C.; PANICHI, V. B. S.; FIGUEIREDO, J. A. S. Risco: a percepção da comunidade ribeirinha do Rio dos Sinos em 
relação ao uso de defensivos agrícolas. Revista Eletrônica em Gestão, educação e tecnologia Ambiental (REGET) - v. 18 n. 2 Mai-Ago. 2014, p.796-802.

SANCHES, S. M. et al. Pesticidas e seus respectivos riscos associados a contaminação da água. Revista de Ecotoxicologia e Meio Ambiente, v. 13, p.53-58. 2003.

SANTOS, W. M. C; ALVES, G. B. M., Modelagem do potencial de poluição hídrica da Bacia Hidrografica do Rio Manso - MT. Brazilian Geographical Journal: Geosciences and Humanities research medium, v. 5, n. 1, p. 289-304, jan./jun. 2014.

SCHEUERMANN, K. K.; EBERHARDT, D. S. Avaliação de fungicidas para o controle da brusone de panícula na cultura do arroz irrigado. Revista de Ciências Agroveterinárias, n. 1, p. 23-28, 2011. ISSN, 16769732.
SERGIPE. SRH - Superintendência de Recursos Hídricos. Atlas digital sobre os Recursos Hídricos de Sergipe. ARACAJU: SEPLANTEC-SRH, CD-ROM. 2012.

SILVA, A. A.; SILVA, J. F. Tópicos em manejo de plantas daninhas. ed. Viçosa: UFV, 2007. cap. 5, p. 189-248.

SILVÉRIO, F. O.; SILVA, J. G. S.; AGUIAR, M. C. S.; CACIQUE, A. P.; PINHO, G. P., Análise de agrotóxicos em água usando extração líquido-líquido com partição em baixa temperatura por cromatografia líquida de alta eficiência, Química Nova vol.35 no.10 São Paulo 2012.

VARGAS, M. A. M. Desenvolvimento regional em questão: O Baixo São Francisco revisitado. ed. São Cristovão: UFS/NPGEO, 1999. 279p. 